\title{
Newly Recognized Pediatric Cases of Typhus Group Rickettsiosis, Houston, Texas, USA
} \begin{abstract}
MedSCape ACTIVITY
In support of improving patient care, this activity has been planned and implemented by Medscape,
LLC and Emerging Infectious Diseases. Medscape, LLC is jointly accredited by the Accreditation Council
for Continuing Medical Education (ACCME), the Accreditation Council for Pharmacy Education (ACPE),
and the American Nurses Credentialing Center (ANCC), to provide continuing education for the
healthcare team.
Medscape, LLC designates this Journal-based CME activity for a maximum of 1.00 AMA PRA
\end{abstract}
Release date: November 16, 2017; Expiration date: November 16, 2018

\section{Learning Objectives}

Upon completion of this activity, participants will be able to:

- Evaluate clinical features of cases of typhus group rickettsiosis (TGR) at a large, Houston-area pediatric hospital between 2008 and 2016, based on a retrospective medical record review

- Compare epidemiological features of cases of TGR at a large, Houston-area pediatric hospital between 2008 and 2016 , based on a retrospective medical record review

- Assess the public health implications of cases of TGR at a large, Houston-area pediatric hospital between 2008 and 2016, based on a retrospective medical record review

\section{CME Editor}

Dana C. Dolan, BS, Copyeditor, Emerging Infectious Diseases. Disclosure: Dana C. Dolan, BS, has disclosed no relevant financial relationships.

\section{CME Author}

Laurie Barclay, MD, freelance writer and reviewer, Medscape, LLC. Disclosure: Laurie Barclay, MD, has disclosed the following relevant financial relationships: owns stock, stock options, or bonds from Alnylam; Biogen; Pfizer.

\section{Authors}

Disclosures: Timothy Erickson, MSPH; Juliana da Silva, MD; Melissa S. Nolan, MPH, PhD; Flor M. Munoz, MD; and Kristy O. Murray, DVM, PhD, have disclosed no relevant financial relationships. Lucila Marquez, MD, MPH, has disclosed the following relevant financial relationship: received grants for clinical research from Cempra.

\section{Timothy Erickson, Juliana da Silva, Melissa S. Nolan, Lucila Marquez, Flor M. Munoz, Kristy O. Murray}

An increase in typhus group rickettsiosis and an expanding geographic range occurred in Texas, USA, over a decade. Because this illness commonly affects children, we retrospectively examined medical records from 2008-2016 at a large Houston-area pediatric hospital and identified 36 cases. The earliest known cases were diagnosed in 2011.

Author affiliations: The University of Texas Health Science Center, Houston, Texas, USA (T. Erickson); Baylor College of Medicine and Texas Children's Hospital, Houston (T. Erickson, J. da Silva, M.S. Nolan, L. Marquez, F.M. Munoz, K.O. Murray)

DOI: https://doi.org/10.3201/eid2312.170631
Typhus group rickettsiosis (TGR) is a vectorborne zoonotic disease most commonly caused by the bacteria Rickettsia typhi, the etiologic agent of murine typhus. Since 2003, the annual incidence of TGR cases in Texas has increased and expanded geographically (1). Recently, a study conducted in Galveston County found that 7 of 18 persons with acute fever were positive for $R$. typhi (2). In the Houston/Harris County metropolitan area, which is adjacent to Galveston County, TGR was first reported to the Texas Department of State Health Services (TXDSHS) in 2007 (1). By 2013, 27 cases had been reported to the state. Because the highest attack rate occurs in children 5-19 years of age, we became concerned that children with possible $R$. typhi infection were brought to Texas Children's Hospital (TCH), a large, 692-bed pediatric 
Table. Demographic, social, and clinical characteristics of pediatric typhus group rickettsiosis case-patients, Houston, TX, USA, 2008-2016*

\begin{tabular}{|c|c|c|c|c|}
\hline Characteristic & All cases, $n=36$ & Confirmed cases, $n=18$ & $\begin{array}{c}\text { Probable cases, } \\
n=13\end{array}$ & $\begin{array}{c}\begin{array}{c}\text { Suspected } \\
\text { cases, } n=5\end{array} \\
\end{array}$ \\
\hline Median age (range), y & $11(2-23)$ & $9(2-23)$ & $9(4-17)$ & $16(8-19)$ \\
\hline \multicolumn{5}{|l|}{ Sex } \\
\hline$M$ & $18(50)$ & $7(39)$ & $7(54)$ & $4(80)$ \\
\hline $\mathrm{F}$ & $18(50)$ & $11(61)$ & $6(46)$ & $1(20)$ \\
\hline \multicolumn{5}{|l|}{ Race/ethnicity } \\
\hline White & $19(53)$ & $8(44)$ & $9(69)$ & $2(40)$ \\
\hline Hispanic & $15(42)$ & $9(50)$ & $4(31)$ & $2(40)$ \\
\hline Other or unknown & $2(6)$ & $1(6)$ & & $1(20)$ \\
\hline \multicolumn{5}{|l|}{ Exposures } \\
\hline Dogs & $17(47)$ & $9(50)$ & $8(62)$ & 0 \\
\hline Cats & $16(44)$ & $8(44)$ & $6(46)$ & $2(40)$ \\
\hline Opossums & $2(6)$ & $1(6)$ & 0 & $1(20)$ \\
\hline Fleas & $8(22)$ & $4(22)$ & $3(23)$ & $1(20)$ \\
\hline \multicolumn{5}{|l|}{ History of travel } \\
\hline To endemic area of Texas & $4(11)$ & $4(22)$ & 0 & 0 \\
\hline To endemic area outside United States & $2(6)$ & $2(11)$ & 0 & 0 \\
\hline To area with no known Rickettsia typhi & $4(11)$ & $3(17)$ & $1(8)$ & 0 \\
\hline \multicolumn{5}{|l|}{ Signs and symptoms } \\
\hline Fever & $35(97)$ & $17(94)$ & $13(100)$ & $5(100)$ \\
\hline Rash & $26(72)$ & $13(72)$ & $10(77)$ & $3(60)$ \\
\hline Headache & $14(39)$ & $8(44)^{\prime}$ & $3(23)$ & $3(60)$ \\
\hline Malaise & $13(36)$ & $8(44)$ & $5(39)$ & $2(40)$ \\
\hline Vomiting & $12(33)$ & 7 (39) & $4(31)$ & $1(20)$ \\
\hline Anorexia & $11(31)$ & 7 (39) & $4(31)$ & 0 \\
\hline Classical triad $\dagger$ & $11(31)$ & $5(28)$ & $3(23)$ & $3(60)$ \\
\hline Lymphadenopathy & $10(28)$ & $4(22)$ & $4(31)$ & $2(40)$ \\
\hline Abdominal pain & $10(28)$ & $5(28)$ & $4(31)$ & $1(20)$ \\
\hline Conjunctivitis & $9(25)$ & $4(22)$ & $4(31)$ & $1(20)$ \\
\hline \multicolumn{5}{|l|}{ Clinical findings } \\
\hline Hepatosplenomegaly & $6(19)$ & $4(22)$ & $2(15)$ & $1(20)$ \\
\hline Altered mental status & $3(8)$ & $3(17)$ & 0 & 0 \\
\hline \multicolumn{5}{|l|}{ Elevated aminotransaminases } \\
\hline Aspartate aminotransaminase & $31(86)$ & $16(89)$ & $11(85)$ & $4(80)$ \\
\hline Alanine aminotransaminase & $32(89)$ & $17(94)$ & $11(85)$ & $4(80)$ \\
\hline Hypoalbuminemia & $11(31)$ & $6(33)$ & $3(23)$ & $2(40)$ \\
\hline Thrombocytopenia & $22(61)$ & $11(61)$ & $9(69)$ & $2(40)$ \\
\hline Median days hospitalized (range) & $5(0-14)$ & $6(1-14)$ & $5(0-10)$ & $4(0-5)$ \\
\hline PICU admissions & $7(19)$ & $5(28)$ & $2(15)$ & 0 \\
\hline Median titer for R. typhi (range) & $1: 1,024(1: 64-16,384)$ & $1: 2,048(1: 1,024-1: 16,384)$ & $1: 256(1: 128-1: 512)$ & $1: 64(1: 64)$ \\
\hline \multicolumn{5}{|c|}{$\begin{array}{l}\text { *Values are no. (\%) except as indicated. A confirmed case was defined by one of the following: 1) immunofluorescent antibody (IFA) assay titer } \geq 1: 1,024, \\
\text { and a titer for } R \text {. typhi } \geq 2 \text {-fold greater than that for } R \text {. rickettsii to rule out cross-reactivity; } 2 \text { ) PCR positive; or } 3 \text { ) } \geq 4 \text {-fold increase in titer between acute } \\
\text { and convalescent specimens. A probable case was defined as } 1 \text { ) an IFA titer } \geq 1: 128 \text {, and a titer for } R \text {. typhi } \geq 2 \text {-fold greater than } R \text {. rickettsii and } 2 \text { ) } \\
\text { clinically compatible illness involving fever with rash. A suspected case was defined as } 1 \text { ) IFA titer } \geq 1: 64 \text { and negative titer for } R \text {. rickettsii and } 2 \text { ) clinically } \\
\text { compatible illness. } \\
\dagger \text { Symptoms were fever, headache, and rash. }\end{array}$} \\
\hline
\end{tabular}

hospital system in Houston. To evaluate this recent emergence, we retrospectively searched the hospital diagnostic testing database to identify all TGR-positive patients.

\section{The Study}

We conducted a retrospective review to identify TGR patients seen at TCH from January 1, 2008, through December 31,2016. We identified cases by searching all laboratory orders for rickettsial panel immunofluorescent antibody (IFA) testing. We determined these patients' case status by following guidance from TXDSHS (1). We defined a confirmed case as 1 of the following: 1) IFA assay titer $\geq 1: 1024$ and a titer for $R$. typhi $\geq 2$-fold greater than that for $R$. rickettsii; 2 ) a positive PCR result; or 3 ) a $\geq 4$-fold increase in titer between acute and convalescent specimens. We defined a probable case as IFA titer $\geq 1: 128$ and a titer for $R$. typhi $\geq 2$-fold greater than that for $R$. rickettsii; and a clinically compatible illness involving fever with rash. We defined a suspected case as IFA titer $\geq 1: 64$ and negative titer for $R$. rickettsii; and clinically compatible illness. We abstracted demographic and clinical data on all identified TGR case-patients.

When searching diagnostic laboratory orders for rickettsial IFA, we identified 425 test submissions. On the basis of diagnostic results and clinical compatibility, we identified 36 TGR cases: 18 confirmed, 13 probable, and 5 suspected cases. One case was additionally confirmed $R$. typhi-positive by PCR at the Centers for Disease Control 
and Prevention (Atlanta, GA, USA). Only 3 case-patients had convalescent specimens collected, and each yielded $\geq 4$-fold increase in titer, thereby confirming infection.

The case population was predominantly non-Hispanic whites $(53 \%)$ (Table), which were overrepresented when compared with the proportion of $31 \%$ from the Houston/ Harris County US Census population for mid-2016 (3). Hispanics represented $42 \%$ of the patient population, which matched the known census population (42\%) The black population was underrepresented $(0 \%$ of patients vs. $20 \%$ Census population) (3).

Upon review of the medical records, nearly all casepatients $(35 / 36 ; 97 \%)$ were febrile when they sought care (median temperature $103^{\circ} \mathrm{F}$ ) (Table). Eleven $(31 \%)$ casepatients had the classical triad for typhus of fever, rash, and headache. Other common symptoms included malaise, vomiting, anorexia, abdominal pain, lymphadenopathy, and conjunctivitis. Approximately one fifth had hepatosplenomegaly noted on physical exam. Most case-patients also had elevated transaminases $(86 \%)$ and thrombocytopenia (61\%). A median of 8 days elapsed between symptom onset and arrival at the hospital for medical care and 13 days from symptom onset to defervescence. Whereas the median time from symptom onset to initiation of treatment was 12 days, the median time from initiation of doxycycline treatment to defervescence was only 1 day.

Most case-patients (30/36; 83\%) were admitted for hospitalization, with a median length of stay of 5 days. Of those, 7 (19\%) required intensive care due to severity of illness. Hispanic patients were significantly more likely to be admitted to the pediatric intensive care unit $(86 \%$ of these patients were Hispanic; odds ratio $12.7,95 \%$ CI 1.2-612.3; $p=0.027$ by 2-tailed Fisher exact test). No deaths occurred.

All but 2 case-patients had reported animal exposure, and $27(75 \%)$ had reported exposure to either domestic
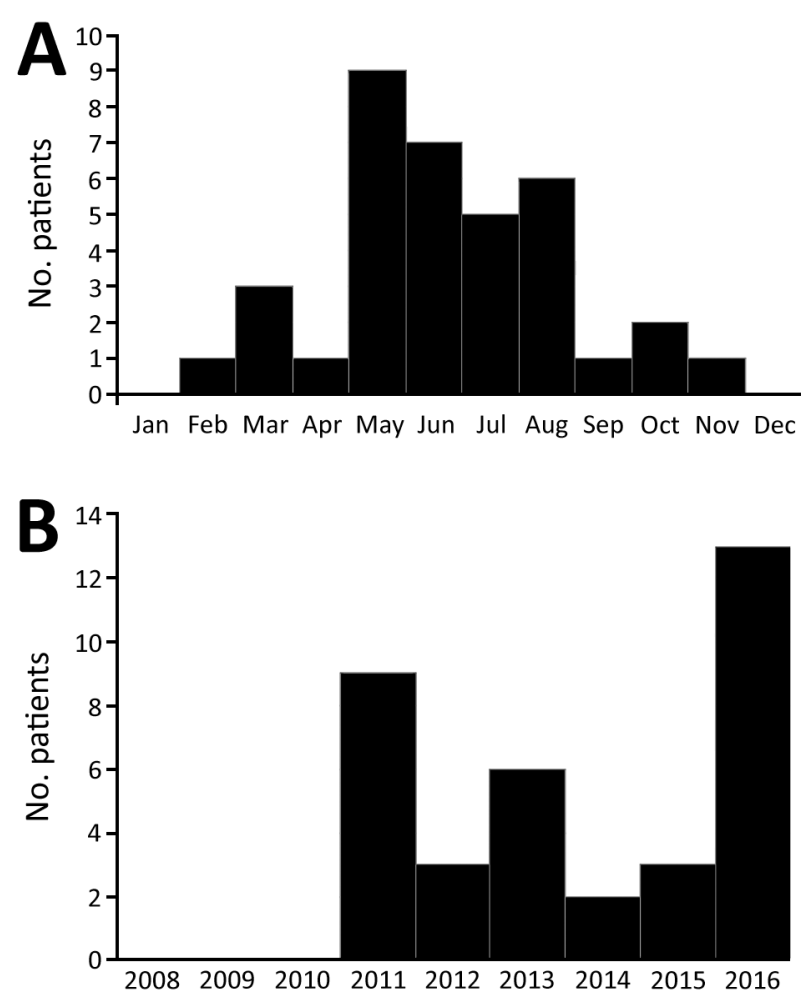

Figure 1. Temporal distribution of Rickettsia typhi-positive pediatric case-patients by time of symptom onset, Houston, Texas, USA, 2011-2016. A) By month of symptom onset. B) By year of symptom onset.

dogs or cats. Two case-patients reported contact with an opossum; 1 of these had no contact with domestic pets. Eight case-patients reported contact with fleas, and all 8 also reported animal contact. Most cases were reported during summer months when fleas are most prevalent (Figure 1, panel A). No TGR cases of were diagnosed in

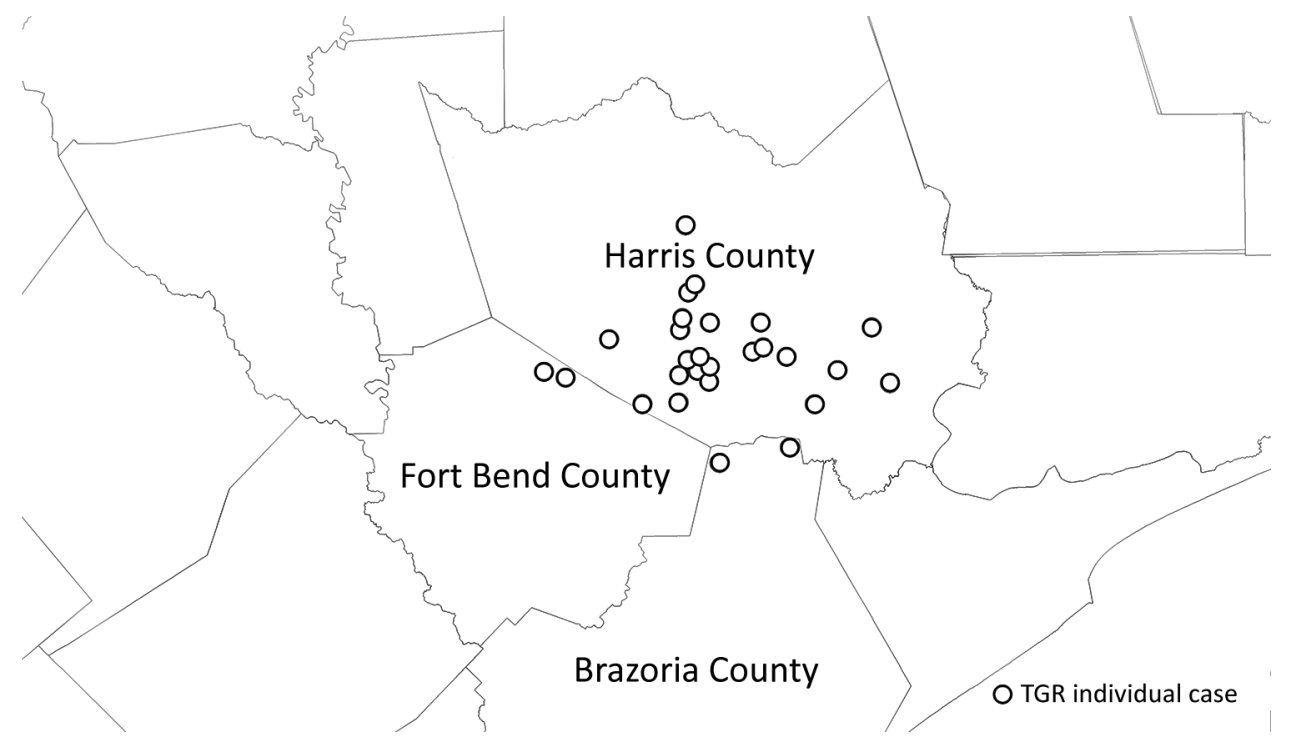

Figure 2. Spatial distribution of Rickettsia typhi-positive pediatric case-patients with no history of travel by location of residence around the Houston/ Harris County, Texas, USA, metropolitan region. 
2008-2010. The year with the highest number of cases was 2016 (Figure 1, panel B).

Thirty $(83 \%)$ case-patients resided in the Houston metropolitan area; cases were geographically clustered in western Houston (Figure 2). Of these case-patients, 26 (72\%) had no history of travel. Three cases were within 70 miles of the Louisiana border. In Louisiana, TGRs are not reportable diseases (4).

Recently, we demonstrated evidence of TGR reemergence into new geographic areas of Texas, including Houston/Harris County, the third most populated county in the United States (1). Here we present the clinical findings of pediatric patients with TGRs in the Houston metropolitan area, starting in 2011. To track this evidence of emergence, it is critical to raise clinical awareness and encourage testing, diagnosis, and public health reporting of new cases.

Rickettsial infections are reportable in Texas, with cases passively reported by medical care providers. In working with TXDSHS, we cross-referenced our patient list with the public health surveillance database and found only 15 (48\%) of the 31 confirmed or probable cases were reported, highlighting a critical gap in passive surveillance. Reporting did improve over time, with 71\% (10/14) of 2015-2016 cases reported to TXDSHS, compared with only $29 \%(5 / 17)$ of 2011-2014 cases. Barriers to reporting could be related to low awareness of reporting requirements, complexity of patient care, and the inherent delays in receiving testing results, typically after discharge. Public health surveillance is critical for disease tracking, prevention, and control efforts; therefore, further work is needed to optimize public health reporting of rickettsial infections.

Approximately one third of the pediatric TGR cases we report exhibited the classic triad of fever, headache, and rash, which is considered the hallmark of $R$. typhi infection. Another pediatric study also reported similar findings (5). The severity of illness in our patients was remarkable, with 1 in 5 patients requiring intensive care. Early clinical suspicion, diagnosis, and appropriate treatment of suspected rickettsial infections is critical to shorten the duration of illness and prevent serious, life-threatening outcomes.

Our study documents the identification and clinical description of pediatric cases of TGRs in the Houston area. While we believe TGR is emerging locally, it is plausible that prior cases simply went undetected and undiagnosed. A study conducted in 2004 found that $10 \%$ of homeless persons in Houston tested seropositive for R. typhi (6). That study looked only at past exposure, however, so we do not know where those persons acquired the infection. Because studies are lacking in this region regarding the specific reservoirs and vectors responsible for transmission, establishing research in this area is critical.

\section{Conclusions}

The recent emergence of TGRs in Houston poses a public health threat. Our report provides insight into the presentation and epidemiology of disease in a pediatric population. It is still unknown what factors put these children at risk for infection. The sylvatic and domestic transmission cycles of $R$. typhi, including vectors and mammalian reservoirs, require further investigation. Improved physician awareness through reporting of clinical studies and case series will assist in appropriate diagnosis and management of disease throughout Texas and the southern United States.

\section{Acknowledgments}

We thank Bonny Mayes for assisting with cross-referencing our cases with the state surveillance database.

This study was reviewed and approved by the Baylor College of Medicine Institutional Review Board (H-34878).

Mr. Erickson is a doctoral student in epidemiology at the University of Texas Health Science Center at Houston, School of Public Health. He received his MSPH in public health from Texas A\&M University. His research interests include the epidemiology of infectious diseases, particularly rickettsial diseases.

\section{References}

1. Murray KO, Evert N, Mayes B, Fonken E, Erickson T, Garcia MN, et al. Typhus group rickettsiosis, Texas, USA, 2003-2013. Emerg Infect Dis. 2017;23:645-8. http://dx.doi.org/10.3201/eid2304.160958

2. Blanton LS, Vohra RF, Bouyer DH, Walker DH. Reemergence of murine typhus in Galveston, Texas, USA, 2013. Emerg Infect Dis. 2015;21:484-6. http://dx.doi.org/10.3201/eid2103.140716

3. US Census Bureau. Quick facts: Harris County, Texas. 2015 [cited 2016 Aug 1]. http://www.census.gov/quickfacts/table/ AGE275210/48201

4. Louisiana Department of Health. Sanitary code - state of Louisiana. Part 2 - the control of disease. 2016 [cited 2017 Sep 26] http://new.dhh.louisiana.gov/assets/oph/Center-PHCH/Center-CH/ infectious-epi/Surveillance/sanitarycode.pdf.

5. Whiteford SF, Taylor JP, Dumler JS. Clinical, laboratory, and epidemiologic features of murine typhus in 97 Texas children. Arch Pediatr Adolesc Med. 2001;155:396-400. http://dx.doi.org/ 10.1001/archpedi.155.3.396

6. Reeves WK, Murray KO, Meyer TE, Bull LM, Pascua RF, Holmes KC, et al. Serological evidence of typhus group rickettsia in a homeless population in Houston, Texas. J Vector Ecol. 2008;33:205-7. http://dx.doi.org/10.3376/ 1081-1710(2008)33[205:SEOTGR]2.0.CO;2

Address for correspondence: Kristy O. Murray, Baylor College of Medicine, 1102 Bates Ave, Ste 550, Houston, TX 77030, USA; email: kmurray@bcm.edu 